\title{
Semiconductor Spintronics: Role of the Valence-Band Holes
}

\begin{abstract}
A. DARGYS*
Semiconductor Physics Institute, A. Goštauto 11, 01108 Vilnius, Lithuania

Two aspects related to valence-band hole spin are considered: spin surfaces in $p$-type semiconductors and flipping of the hole spin by an ultrashort electric field pulse. It is shown that heavy, light, and split-off holes have different spin surfaces. In general, the shape of the surface in real semiconductors may depend on the hole wave vector direction and magnitude. The concept of spin surface is used to explain very strong anisotropy of hole spin injection efficiency observed recently in ferromagnetic-semiconductor structures and in optimizing ultrafast spin switching. It was shown that, of all spin flipping mechanisms, the most effective one is associated with hole transfer between different spin surfaces in high electric fields. The less effective mechanisms are related to valence band warping and nonparabolicity. Examples of the hole spin flipping dynamics and the discussion on ultrafast control of spin in semiconductors by $\pi$-type electrical pulses are presented.
\end{abstract}

PACS numbers: 72.20.Jv, 73.40.Gk, 78.55.-m, 79.90.+b

\section{Introduction}

The subject of spintronics, including semiconductor spintronics, where both electron spin and charge are important in the operation of solid-state devices, was discussed recently in the review by Žutić et al. [1]. From the review it follows that experimental and theoretical investigations are mainly devoted to electron spin. The interest in the electron spin in semiconductors partly may be explained by long memory: normally the spin memory is lost after hundred or more wave vector randomizing electron collisions. The spin memory of the hole, due to strong spin-orbit interaction in the valence band, is much shorter. On the other hand, a complex energy spectrum of the valence band gives richer and deeper spin physics.

The main difference between spin properties in conduction and valence bands comes from the strength of spin-orbit interaction. The perturbation theory cannot be applied to the analysis of hole spin properties as it is normally done in conduction band spin case [2]. Below we shall show that a concept of the spin surface related to a particular valence band appears to be very helpful in the understanding

*e-mail: dargys@pfi.lt 
hole spin in general, and spin injection and transport properties in particular [3-5]. The latter properties are very important in the applied spintronics and in the optimization of spin flipping transitions by ultrashort electrical pulses [6-10]. The ultrafast switching of spin is a new and exciting area in the spintronics, which may find new applications in bulk and low dimensional semiconductor terahertz electronics.

In the second part of this report, at first, a concept of the spin surface in a simple doubly-degenerate quantum system is introduced and then the spin surfaces in real semiconductors are described. In the third part, using the concept of the spin surface the control of hole spin by electric field is considered.

\section{Spin surfaces}

$$
\text { 2.1. Spin- } \frac{1}{2} \text { case }
$$

The spin surface describes all possible locations of the tip of average spin or total angular momentum of the free charge carrier, when the state vector that represents a given band is parameterized. In the case of the conduction bands, where the energy band minimum is at the center of the Brillouin zone in $\Gamma$ point, the electron spin is described by the Pauli matrices $\left(\sigma_{x}, \sigma_{y}, \sigma_{z}\right) \equiv \boldsymbol{\sigma}$. If $\psi$ is the wave function of the electron, then the average electron spin is half of

$$
\langle\boldsymbol{\sigma}\rangle=\langle\psi|\boldsymbol{\sigma}| \psi\rangle \text {. }
$$

If the wave function is parameterized, for example, in the form $\psi=$ $\left(a, \sqrt{1-a^{2}} \exp (\mathrm{i} \phi)\right)$, where the parameters $a$ and $\phi$ describe the phase relations between and populations of up and down spin states, then one finds from (1) that

$$
\langle\boldsymbol{\sigma}\rangle=\left(2 a \sqrt{1-a^{2}} \cos \phi, 2 a \sqrt{1-a^{2}} \sin \phi,-1+2 a^{2}\right) .
$$

From Eq. (2) follows that in this case $\left\langle\sigma_{x}\right\rangle^{2}+\left\langle\sigma_{y}\right\rangle^{2}+\left\langle\sigma_{z}\right\rangle^{2}=1$ for all $a$ 's and $\phi$ 's. Thus, if Eq. (2) is plotted in three-dimensional spin space one will have a sphere of a unit radius. When the parameters $a$ and $\phi$ are allowed to vary, the tip of the spin vector (2) will trace the geodesic lines on the sphere. The family of all possible lines represents the spin surface. Thus, the spin surface contains all possible outcomes of a measurement of spin direction and magnitude of the considered quantum system. In the following we shall be interested in the spin surfaces which are described by the total angular momentum $\boldsymbol{J}$ of the heavy, light and split-off band holes.

\subsection{Simple valence bands}

Due to strong spin-orbit interaction between valence bands, it appears that the resulting hole spin surfaces are not necessarily spheres. In calculating the shape of surfaces the main difficulty comes from the fact that, even in the lowest order approximation, the valence band Hamiltonian and the total angular momentum operator $\boldsymbol{J}$ do not commute. This means that there is no common basis to 
represent the hole energy and the spin simultaneously, as was the case with the simple half-spin system or $\Gamma$-type conduction band electrons. To connect the spin basis, in which the Luttinger-Kohn Hamiltonian [11] has been constructed, with the parameterized energy basis, where the Hamiltonian is diagonal and describes the dispersion laws of all three valence bands, one must find a unitary transformation that couples mentioned six-component basis vectors. In general case this can be done by numerical methods only. However, when the light and heavy hole energy bands are spherical and parabolic and the split-off band can be neglected, it appears possible to find such unitary transformation matrix $\mathcal{T}$. If the parameterized form of the heavy, or light band wave function in the energy representation is $|f\rangle$, and the same function in the spin representation is $|\psi\rangle$, then $|\psi\rangle=\mathcal{T}|f\rangle$. It will be assumed that the parameterized forms of the heavy and light hole wave functions can be written in the following way:

$$
\begin{aligned}
& \left|f_{\mathrm{h}}\right\rangle=\left(\cos \theta_{\mathrm{h}}, \mathrm{e}^{\mathrm{i} \phi_{\mathrm{h}}} \sin \theta_{\mathrm{h}}, 0,0\right), \\
& \left|f_{\mathrm{l}}\right\rangle=\left(0,0, \cos \theta_{\mathrm{l}}, \mathrm{e}^{\mathrm{i} \phi_{\mathrm{l}}} \sin \theta_{\mathrm{l}}\right) .
\end{aligned}
$$

Then, the corresponding spin surfaces for heavy and light holes having the wave vector $\boldsymbol{k}=\left(0,0, k_{z}\right)$ are described by the geodesic lines [5]:

$$
\begin{aligned}
\langle\boldsymbol{J}\rangle_{\mathrm{h}} & =\left(0,0, \frac{3}{2} \cos \phi_{\mathrm{h}} \sin 2 \theta_{\mathrm{h}}\right), \\
\langle\boldsymbol{J}\rangle_{\mathrm{l}} & =\left(-\sin \phi_{\mathrm{l}} \sin 2 \theta_{\mathrm{l}}, \cos 2 \theta_{\mathrm{l}},-\frac{1}{2} \cos \phi_{\mathrm{l}} \sin 2 \theta_{\mathrm{l}}\right) .
\end{aligned}
$$

Here $\langle\boldsymbol{J}\rangle_{\mathrm{h}, 1}$ is measured in units of $\hbar$. Figure 1a and b shows the lines (5)-(6) in $3 \mathrm{D}$ spin space, when one of the parameters, $\theta_{\mathrm{h}, 1}$ or $\phi_{\mathrm{h}, \mathrm{l}}$, is varied. Figure $1 \mathrm{c}$ shows the spin surface of the split-off band, which appears to be a sphere of radius $\left|\langle\boldsymbol{J}\rangle_{\mathrm{s}}\right|=1 / 2$. The latter was calculated using $6 \times 6$ Hamiltonian for small wave vectors [5]. The following points are worth mentioning in connection with Fig. 1.
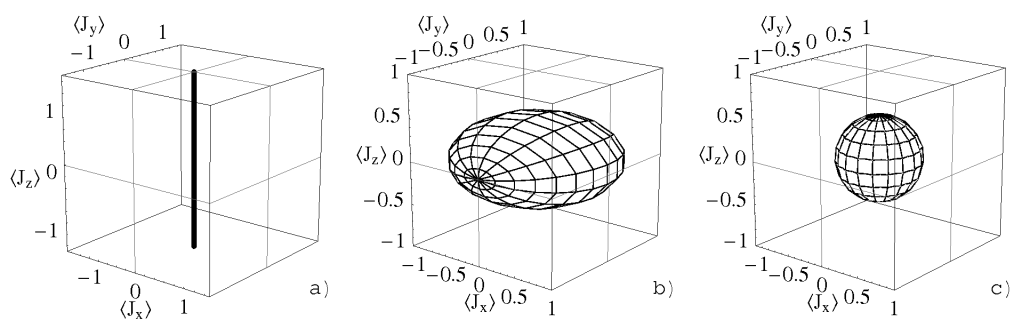

Fig. 1. Spin surfaces of (a) heavy, (b) light, and (c) split-off bands in the spherical and parabolic energy band approximation. The wave vector $\boldsymbol{k}$ is parallel to $\left\langle J_{z}\right\rangle$. For other directions of $\boldsymbol{k}$ the surfaces should be rotated in the same way as $\boldsymbol{k}$. 
The surfaces in Fig. 1 represent all possible outcomes of the hole spin measurement, when the hole is in one of the bands (heavy, light, or split-off) and propagates in $z$ direction, i.e., along the quantization axis. If hole wave vector $\boldsymbol{k}$ is pointing in a different direction, the spin surfaces in Fig. 1 should be rotated from the $z$ direction in the same way as $\boldsymbol{k}$. As seen from Eqs. (5) and (6), the spin surfaces are independent of wave vector magnitude in this simple case. Thus, in the spherical and parabolic band approximation the spin of the heavy hole reduces to a line parallel to $\boldsymbol{k}$. Depending on parameter $\theta_{\mathrm{h}}$ and $\phi_{\mathrm{h}}$, the length of $\left|\langle\boldsymbol{J}\rangle_{\mathrm{h}}\right|$ can assume any value between $-3 / 2$ and $3 / 2$. The spin surface of the light hole is an oblate ellipsoid of revolution, Fig. $1 \mathrm{~b}$, with the minor axis equal to $1 / 2$. The major axes are perpendicular to $\boldsymbol{k}$ and are equal to 1. Figure 1 also represents the properties of an intrinsic hole spin $\langle\boldsymbol{S}\rangle$, since in this approximation the $4 \times 4$ matrices of the total angular momentum $\boldsymbol{J}$ and the intrinsic spin $\boldsymbol{S}$, apart from a numerical factor 3, coincide [12]. In conclusion, even in the simple band model, where bands are spherical, parabolic, and doubly degenerate, one finds that, contrary to conduction band electron, the heavy and light hole spin surfaces are different from sphere. This may give interesting effects in the spin transport and injection properties.

Recently, it was observed that the injection efficiency of hole spins differs markedly when the hole spin is parallel and perpendicular to the injection plane in the ferromagnetic-semiconductor structure [13]. This effect finds a simple explanation in the spin surface parlance, if one remembers that the heavy hole spin is always parallel to hole propagation direction. Since the injected holes move away from the ferromagnetic-semiconductor interface their spins will predominantly be directed along the current. When the hole spins in the injector, for example by magnetic field, are aligned along the ferromagnetic-semiconductor interface the hole movement into the interior of the semiconductor will be damped, since inside the semiconductor the heavy hole does not support states where $\langle\boldsymbol{J}\rangle$ and $\boldsymbol{k}$ are perpendicular. As Fig. 1b shows, the effect of anisotropy for light holes has an opposite sign, however, due to much larger density of states in the heavy-mass band, the heavy hole spin injection current will prevail over light hole current and will, as a result, be responsible for the observed spin injection anisotropy in the experiment [13].

\subsection{Degenerate but nonspherical-nonparabolic bands}

In this approximation, hole spin properties were found to be very close to those of elementary and $\mathrm{A}_{3} \mathrm{~B}_{5}$ semiconductors. For diamond-type semiconductors the model is exact, since in this group of materials the spin splitting is absent and, as a result, all bands remain doubly degenerate. The characteristic feature in this approximation is that the shape of spin surfaces becomes dependent on the magnitude of $\boldsymbol{k}$. In Fig. 2, the dependence of $\left\langle\boldsymbol{J}^{2}\right\rangle$ on $|\boldsymbol{k}|$ illustrates a degree of the influence of band nonparabolicity and nonsphericity in case of InP. At small 

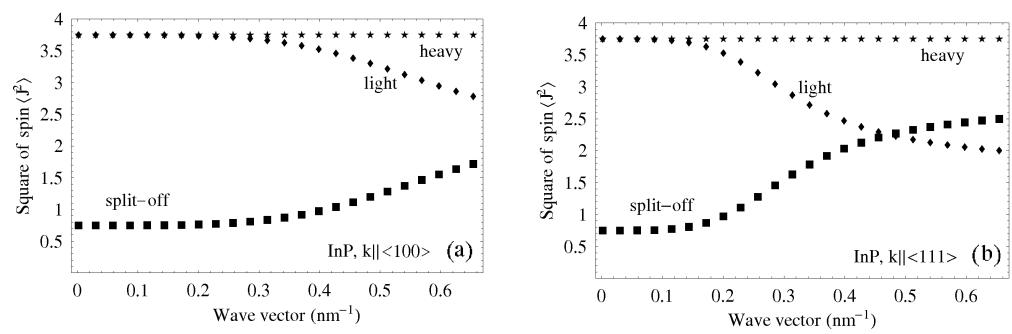

Fig. 2. Dependence of the square of hole spin on the magnitude of hole wave vector $\boldsymbol{k}$ for heavy-mass, light-mass, and split-off bands in InP. (a) $\boldsymbol{k} \|\langle 100\rangle$, (b) $\boldsymbol{k} \|\langle 111\rangle$.
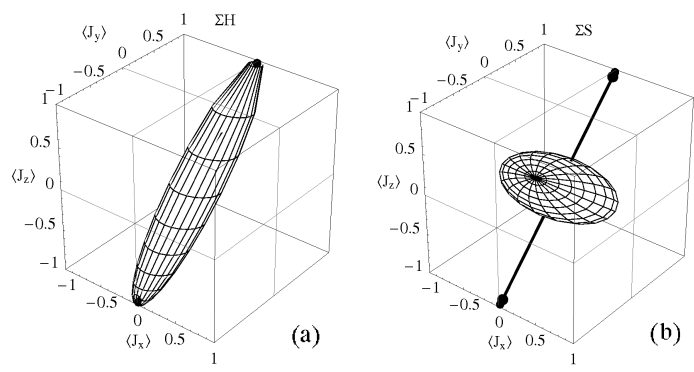

Fig. 3. Spin surfaces in InP at finite wave vector $\boldsymbol{k} \|$ [011] shown by arrows in the part (b). $|\boldsymbol{k}|=0.667 \mathrm{~nm}^{-1}$. (a) Heavy-mass band, (b) split-off band.

wave vectors, when the bands are spherical and parabolic, one has $\left\langle\boldsymbol{J}^{2}\right\rangle_{\mathrm{h}, \mathrm{l}}=15 / 4$ for heavy and light holes and $\left\langle\boldsymbol{J}^{2}\right\rangle_{\mathrm{s}}=3 / 4$ for split-off holes. However, at thermal wave vectors, due to strong mixing of light and split-off bands, the deviation is large and strongly anisotropic. Figure 3 illustrates that in this approximation the heavy hole spin surface is cigar- rather than line-shaped. Extensive calculations [5] using valence band parameters of $\mathrm{A}_{3} \mathrm{~B}_{5}$ compounds [14] show that the largest deviation from the simple model occurs when $\boldsymbol{k}$ is parallel to $\langle 011\rangle$ direction, and the smallest deviation occurs for heavy-mass holes with $\boldsymbol{k} \|\langle 001\rangle$ and $\boldsymbol{k} \|\langle 111\rangle$. In these technologically important directions the heavy hole spin surface to a good approximation can be replaced by a line parallel to $\boldsymbol{k}$, even at thermal hole energies.

\subsection{General case}

Because of absence of inversion symmetry in $\mathrm{A}_{3} \mathrm{~B}_{5}$ compounds, the valence band Hamiltonian of these semiconductors also contains linear in wave vector terms. The linear- $\boldsymbol{k}$ terms lift spin degeneracy of all energy bands except the points which lie on the fourth order axis. In the calculations, linear- $\boldsymbol{k}$ part of the Hamiltonian was taken from Ref. [15]. From the numerical calculations presented in $[4,5]$ it follows that the inclusion of linear- $\boldsymbol{k}$ terms gives, in general, negligible effect on the shape of the spin surfaces in all $\mathrm{A}_{3} \mathrm{~B}_{5}$ semiconductors.

From the present study of hole spin surfaces in diamond-type and $\mathrm{A}_{3} \mathrm{~B}_{5}$ semiconductors [3-5] it can be concluded that the strongest influence on the form 
of the idealized spin surfaces represented in Fig. 1 comes from the valence band nonparabolicity and nonsphericity.

\section{Coherent control of hole spin}

In case of the electron, coherent control of the spin takes place on the spin surface having spherical shape, best exemplified in the spin-echo experiments with an ensemble of the half-spin electrons. In case of the valence bands one has three closely-spaced spin surfaces of different shapes. Thus, in addition to the well-known spin control methods, where specially arranged sequences of the magnetic field pulses are used to force the end of the average spin to draw intricate trajectories on a single spin surface, now a new spin quantum control method based on transitions between different spin surfaces in electric fields is possible. Since the intervalence transitions can be brought about by hole tunneling in high electric fields, the control of the spin in this case can be very fast, of the order of inverse of the interband energy. If the control is performed on a single spin surface, the spin flipping time will be long, since the shortest time is determined by the inverse of the spin splitting energy, which is about few or few tens of meV. Thus an ultrafast switching in the subpicosecond range can be achieved in the hole interspin surface transitions. Since the magnetic field is more inertial than electric field, the use of the electric field will be preferable in the ultrafast spintronics from the practical considerations as well.

The spin dynamics and intervalence transitions were simulated by the following Schrödinger system of equations for the six-component spinor $|\psi\rangle$ in the effective mass approximation:

$$
\mathrm{i} \hbar \frac{\partial|\psi\rangle}{\partial t}=\left[H_{\mathrm{LK}}(\boldsymbol{k})+H_{\mathrm{F}}(\boldsymbol{k})\right]|\psi\rangle,
$$

where $\mathrm{i}=\sqrt{-1}$ and $\hbar$ is the Planck constant. $H_{\mathrm{LK}}(\boldsymbol{k})$ is the $6 \times 6$ Luttinger-Kohn Hamiltonian [11]. $H_{\mathrm{F}}(\boldsymbol{k})$ is the field term

$$
H_{\mathrm{F}}(\boldsymbol{k}) \approx \frac{e \boldsymbol{F}(t)}{\mathrm{i}} \frac{\partial}{\partial \boldsymbol{k}},
$$

where $e$ is the elementary charge. The time dependence of the average spin projections was found from

$$
\left\langle J_{j}\right\rangle_{i}=\left\langle\psi_{i}\left|J_{j}\right| \psi_{i}\right\rangle
$$

where $J_{j}$ is $j$-th component $(x, y$, or $z$ ) of the total $6 \times 6$ angular momentum matrix. The subscript $i$ denotes the band ( $i=\mathrm{h}, \mathrm{l}$, or s). The concrete forms of the matrices $J_{j}$ can be found in [3]. The numerical complex singular-value decomposition was used to find the unitary matrix $\mathcal{T}$ that connects the state vector $|\psi\rangle$ in spinor representation and its partner $|f\rangle$ in the energy band representation: $|\psi\rangle=\mathcal{T}|f\rangle$. 

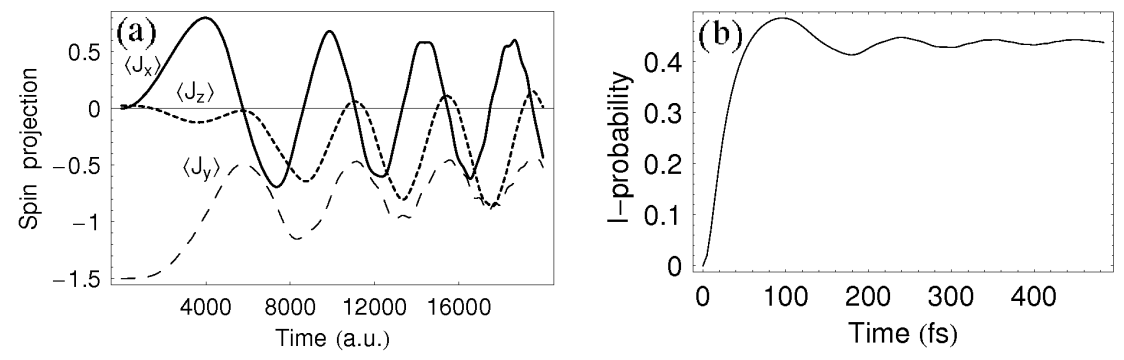

Fig. 4. Spin precession induced by $20 \mathrm{kV} / \mathrm{cm}$ field step switched at the moment $t=0$ (a) and the time-dependence of the probability to find the hole in the light-mass band of Si (b). Initial hole wave vector is $(0,0.236,0) \mathrm{nm}^{-1}, \boldsymbol{F} \| k_{z} .20000$ a.u. $=483 \mathrm{fs}$.

Figure 4a shows the hole spin precession in Si induced by a step of electric field of amplitude $20 \mathrm{kV} / \mathrm{cm}$, that was switched on at the moment $t=0$. The initial spin $\langle\boldsymbol{J}\rangle_{\mathrm{h}}=(0,-3 / 2,0)$, which also represents one of the heavy-hole eigenvalues, was optimized by preselecting the parameters $\theta_{\mathrm{h}}$ and $\phi_{\mathrm{h}}$ in the initial spinor $|f\rangle$. In the experiment this may be achieved by selecting an appropriate spin injector. Figure $4 \mathrm{~b}$, where the probability to find the hole in the light-mass band as a function of time is plotted, explains the origin of spin precession. The figure shows that strong intervalence tunneling is induced at first moments, when energetic distance between heavy- and light-mass bands is not too large. At later moments the probabilities to find the hole in either heavy- or light-mass bands are approximately equal. As a result, the strong beating between heavy and light band wave function is also reflected in the spin component oscillations. Thus, the precession mechanism of the hole spin in the valence band case is different from that for half-spin electron, where the precession is related with the circular motion of the tip of spin vector on a single spin surface. It should be pointed out that in a more general case the band degeneracy will be lifted, so that the slow spin motion on the single surface will also be possible, however, on a much longer time scale. Therefore, a beating between intraspin and interspin surface motions is expected.

Figure 5a shows time dependence of the probability to find the hole in the heavy- and light-mass bands of Si, when the hole is excited by a circularly polarized $\pi$-type electric pulse having Gaussian envelope. The frequency $\omega$ of the exciting pulse was tuned to heavy-light resonance energy at the initial hole wave vector $\boldsymbol{k}_{0}, \hbar \omega=\varepsilon_{1}\left(\boldsymbol{k}_{0}\right)-\varepsilon_{\mathrm{h}}\left(\boldsymbol{k}_{0}\right)$, and the parameters in the envelope function were optimized so that the final probability of excitation to the light-mass band was equal to 1 . Figure $5 \mathrm{~b}$ shows that under such excitation the component $\left\langle J_{z}\right\rangle$ undergoes smooth transition from $\left\langle J_{z}\right\rangle_{\text {ini }}=-3 / 2$ to $\left\langle J_{z}\right\rangle_{\text {fin }}=-1 / 2$ during approximately one-and-half of a picosecond, and the components $\left\langle J_{x}\right\rangle$ and $\left\langle J_{y}\right\rangle$ are excited only at intermediate times. Similar picture is observed when spin excitation occurs on a single spin surface between two eigenstates. If the sense of rotation of the field was reversed to opposite, the intervalence transitions were not observed, in 

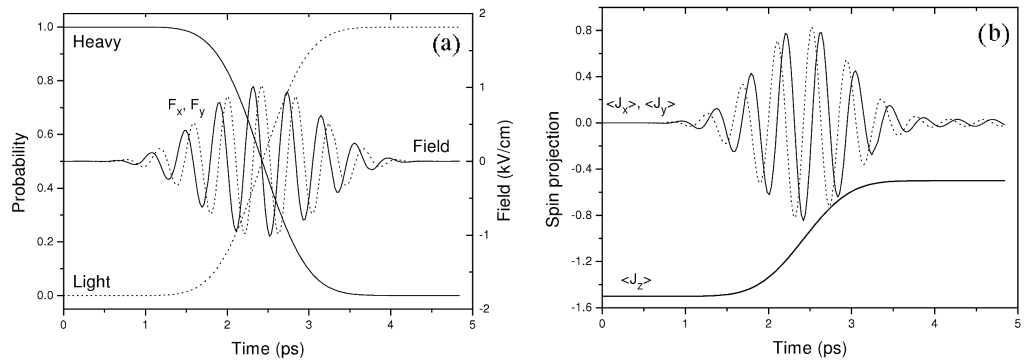

Fig. 5. (a) $F_{x}$ and $F_{y}$ components of the circularly polarized $\pi$-type electric pulse and time dependence of the probability to find the hole in the heavy- and light-mass bands of $\mathrm{Si}$. The frequency is tuned to heavy-light resonance transition, $\hbar \omega=9.89 \mathrm{meV}$. (b) Time dependence of spin components excited by field shown in part (a). The initial hole spin is $\langle\boldsymbol{J}\rangle=(0,0,-3 / 2)$. $\boldsymbol{k}_{0} \|[001]$.
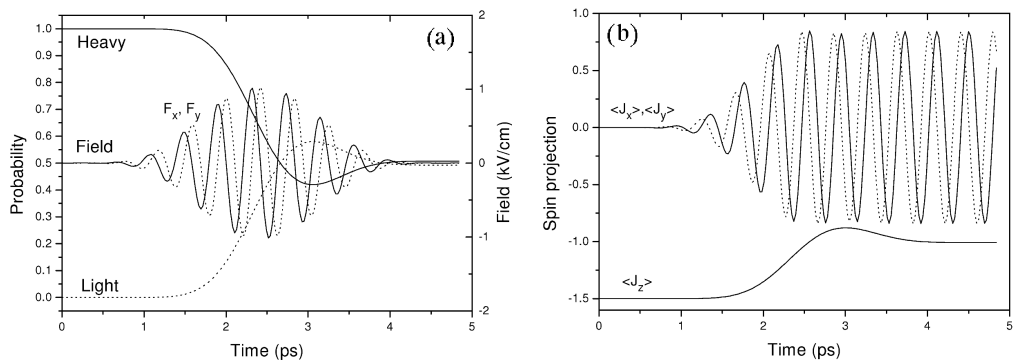

Fig. 6. (a) $F_{x}$ and $F_{y}$ components of the circularly polarized electric field and time-dependence of the probability to find the hole in heavy- and light-mass bands of silicon. The exciting field frequency was detuned by $7.3 \%$ from the resonance frequency $\hbar \omega=$ $9.89 \mathrm{meV}$. (b) Spin components. The initial spin is $\langle\boldsymbol{J}\rangle=(0,0,-3 / 2)$ and $\boldsymbol{k}_{0} \|[001]$. This figure is to be compared with Fig. 5 .

accordance with the well-known spin selection rules for spin transitions. If the excitation frequency is detuned about $7 \%$ from the resonance condition, as Fig. 6 a demonstrates, after the pulse the hole finds itself in the heavy-light superposition state. In the latter case the final $z$ component of the spin is also in the superposition of heavy- and light-hole spins, i.e., it is not described by heavy or light band spin surface only. As a result the average final $z$ component of $\operatorname{spin}$ is $\left\langle J_{z}\right\rangle_{\text {fin }} \approx 1$. It should be noted that the beating between heavy and light mass bands at the end of the pulse is reflected in $\left\langle J_{x}\right\rangle$ and $\left\langle J_{y}\right\rangle$ components.

Figure 7 shows the dependence of the final value of $\left\langle J_{z}\right\rangle$ on the exciting photon energy $\hbar \omega$. The continuous line is approximation with the Lorentzian curve. To show that there is a strong correlation between population and spin excitation, in the same figure the probability to excite the hole to light-mass band after termination of the pulse is plotted by black circles as well. 


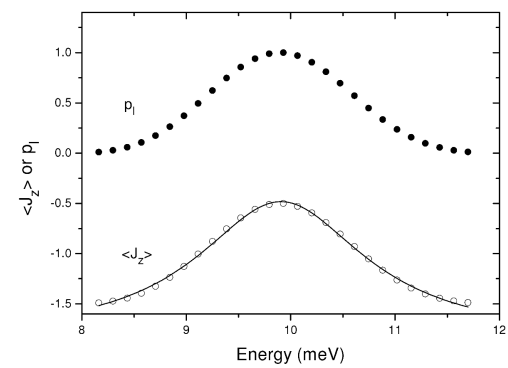

Fig. 7. The final spin projection $\left\langle J_{z}\right\rangle$ and the probability $p_{1}$ to excite light-mass band by $\pi$-type pulse versus photon energy.
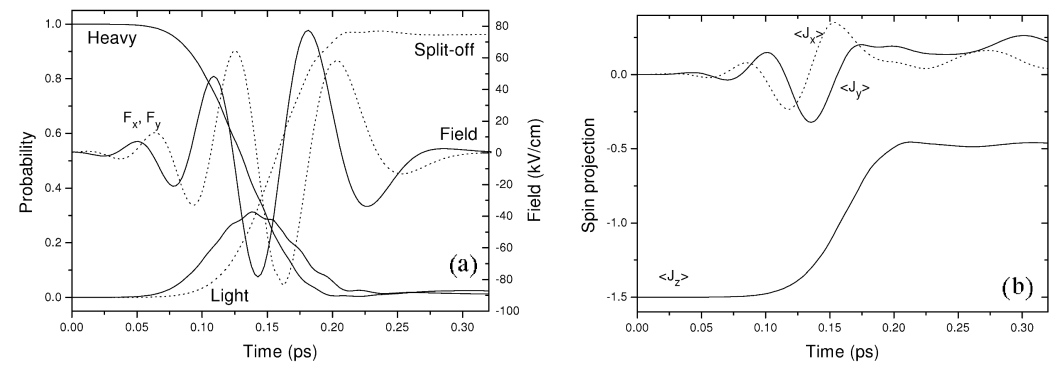

Fig. 8. (a) Ultrashort and optimized heavy-split-off transition induced by $\pi$-type electric field pulse $\left(F_{x}, F_{y}\right)$, and the probabilities to find the hole in the heavy-, light-mass, and split-off bands vs. time. (b) Time-dependence of the spin components. The initial spin is $\langle\boldsymbol{J}\rangle=(0,0,-3 / 2), \boldsymbol{k}_{0} \|[001]$.

The knowledge of spin surfaces and elementary spin excitation processes between surfaces allows one to find optimal initial and final states between which the spin switching occurs in the subpicosecond time scale as Fig. 8 demonstrates. Here, the ultrashort and "circularly" polarized $\pi$-type electric pulse was optimized so as to give maximal excitation of the split-off band and, at the same time, optimal switching of the spin. At $t=0$, the ballistic hole was in the heavy-mass band with $\boldsymbol{k}_{0}=(0,0,0.378) \mathrm{nm}^{-1}$. Since the final excitation probability of the split-off band is somewhat smaller than 1 , one can also see the residual oscillations in the $\left\langle J_{x}\right\rangle$ and $\left\langle J_{y}\right\rangle$ components. Let us note that at intermediate times the light-mass band is excited too, thus, at intermediate times all three valence band spin surfaces take part in the coherent spin switching process in this case.

\section{Conclusions}

The aim of this short review was to show that dynamical, as well as static properties of hole spin are much richer than their energy spectrum properties. It was shown that the concept of spin surface may be very useful in visualizing and finding the initial and final parameterized hole spin states and in describing the spin dynamics in time-dependent electric fields. The most striking property which 
may find application in the semiconductor spintronics is associated with heavy-mass hole. The fact that the heavy-hole spin is directed predominantly along the wave vector may result in interesting spin injection and transport properties, not observed in $n$-type semiconductors. This property is preserved even at high energies, when the bands are nonparabolic and nonspherical, especially when the hole wave vector is pointing in $\langle 001\rangle$ or $\langle 111\rangle$ directions.

Two kinds of coherent control of the hole spin should be distinguished. The first one is connected with the motion of the average spin on one of the spin surface. Such motion is associated with one energy band, too. This kind of the control is similar to that used in the standard EPR spectroscopy, except that the spin surfaces are not spherical in this case. Since the hole spin and the wave vector directions are correlated strongly, the rotation of the wave vector of the ballistic hole by electric field will also induce similar changes in the hole spin. This should be particularly pronounced for heavy-mass holes, the spin surfaces of which are strongly stretched along the wave vector direction. The second and new control method is associated with the intervalence hole transitions. In these transitions, the initial and final spins lie on different spin surfaces. The second method is more perspective in the ultrafast spintronics, since hole intervalence transitions, due to fast interband tunneling in high electric fields, will induce a coherent and ultrafast, shorter than hole collision time with lattice phonons, switching of the hole spin at the same time.

\section{References}

[1] I. Žutić, J. Fabian, S. Das Sarma, Rev. Mod. Phys. 76, 323 (2004).

[2] W. Zawadzki, P. Pfeffer, Semicond. Sci. Technol. 19, R1 (2004).

[3] A. Dargys, Phys. Status Solidi B 241, 145 (2004).

[4] A. Dargys, Lithuanian J. Phys. 44, 267 (2004).

[5] A. Dargys, Phys. Status Solidi B 241, 2954 (2004).

[6] A. Dargys, Phys. Rev. B 66, 165216 (2002).

[7] A. Dargys, P. Harrison, Semicond. Sci. Technol. 18, 247 (2003).

[8] A. Dargys, Phys. Scr. 67, 505 (2003).

[9] A. Dargys, IEEE J. Select. Topics Quant. Electron. 10, 155 (2004).

[10] A. Dargys, Acta Phys. Pol. A 105, 295 (2004).

[11] J.M. Luttinger, W. Kohn, Phys. Rev. 97, 869 (1955).

[12] M. Abolfath, T. Jungwirth, J. Brum, A.H. MacDonald, Phys. Rev. B 63, 054418 (2001).

[13] D.K. Young, E. Johnston-Halperin, D.D. Awschalom, Y. Ohno, H. Ohno, Appl. Phys. Lett. 80, 1598 (2002).

[14] I. Vurgaftman, J.R. Meyer, L.R. Ram-Mohan, J. Appl. Phys. 89, 5815 (2001).

[15] A. Foreman, Phys. Rev. Lett. 86, 2641 (2001). 